Revista Eletrônica de Farmácia

Eletronic Journal of Pharmacy

ISSN 1808-0804

DOI 10.5216/ref.v16.50595

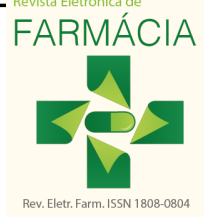

Artigo Original

\title{
Formula® - Sistema web para formulação de cremes e loções
}

\section{Formula $\AA_{-}$- Web system for creams and lotions formulations}

\section{Formula $\AA^{B}$ - Sistema web para la formulación de cremas y lociones}

\author{
DUKIEVICZ, Fábio José ${ }^{1}$. MACHADO, Claudinei José ${ }^{1}$. PAULA, Daniel de ${ }^{2}$. \\ ${ }^{1}$ Faculdade Guairacá - FAG. ${ }^{2}$ Universidade Estadual do Centro-Oeste - Unicentro. \\ *daniel.unicentro@gmail.com
}

Resumo. Introdução: Cremes e loções são os principais veículos de produtos cosméticos e dermatológicos. A formulação desses produtos depende da análise de diversificada e vasta quantidade de informações, dificultando seu aprendizado e geralmente levando a simples reprodução de fórmulas prontas. Objetivo: Desenvolver um sistema Web direcionado a estudantes, profissionais e pesquisadores, que tenha aplicação como ferramenta virtual de ensino-aprendizagem no desenvolvimento de cremes e loções de uso cosmético e dermatológico. Metodologia: O desenvolvimento do software iniciou-se com a definição dos requisitos e funcionalidades mínimas. A validação das fórmulas pelo sistema baseia-se na análise de quinze regras conceituais. O sistema foi criado com o framework PHP Laravel, banco de dados MySQL e as linguagens Cascading Style Sheets (CSS), JavaScript e HTML. Resultados: O sistema possui um banco de dados com informações das substâncias usadas na criação de emulsões e apresenta um mecanismo que permite a sua escolha, de modo a obter a fórmula de uma emulsão. O sistema também é capaz de gerar o método de preparo com base nas propriedades das matérias-primas. Conclusão: $O$ sistema contribui para a redução de custos, uma vez que o usuário irá cometer a maioria dos erros no ambiente virtual sem o dispêndio de matérias-primas e mão de obra.

Palavras-chave: Creme para a Pele. Software. Aprendizagem.

Abstract. Introduction: Creams and lotions are the main vehicles for cosmetic and dermatological products. The formulation of these products depends on the analysis of a diversified and vast amount of information, hindering their learning and generally leading to the simple reproduction of ready-made formulas. Objective: To develop a Web system aimed at students, professionals and researchers that has application as a virtual teaching-learning tool in the development of cosmetic and dermatological creams and lotions. Methodology: Software development began with the definition of minimum requirements and functionalities. The validation of the formulas by the system is based on the analysis of fifteen conceptual rules. The system was created with the PHP Laravel framework, MySQL database and CSS, JavaScript and HTML languages. Results: The system has a database with information of the compounds used in the creation of emulsions and presents a mechanism that allows to choose these ingredients thus forming an emulsion formula. The system is also capable of generating the preparation method based on the properties of the ingredients. Conclusion: The system contributes to the reduction of costs, since the user will commit most errors in the virtual environment without the expense of materials and labor.

Key-words: Skin cream. Software. Learning.

Resumen. Introducción: Las cremas y lociones son los principales vehículos para los productos cosméticos y dermatológicos. La formulación de estos productos depende del análisis de diversa y amplia cantidad de información, lo que dificulta su aprendizaje y generalmente conduce a la simple reproducción de fórmulas pre-elaboradas. Objetivo: Desarrollar un sistema web dirigido a estudiantes, profesionales e investigadores que sea aplicado como herramienta virtual de enseñanza-aprendizaje en el desarrollo de cremas y lociones cosméticas y dermatológicas. Metodología: El desarrollo del software comenzó con la definición de requisitos y funcionalidades mínimas. La validación de las fórmulas se basa en el análisis de quince reglas conceptuales. Fue utilizado el framework PHP Laravel, base de datos MySQL y los lenguajes CSS, JavaScript y HTML. Resultados: El sistema posee una base de datos con información de las sustancias utilizadas en la creación de emulsiones y presenta un mecanismo que permite elegirlas, elaborando de esa manera la fórmula de una emulsión. El sistema también es capaz de generar el método de preparación basado en las propiedades de los ingredientes. Conclusión: El sistema contribuye a la reducción de costos, ya que el usuario cometerá la mayoría de los errores en el entorno virtual sin el gasto de materias primas y mano de obra.

Palabras-clave: Crema para la piel. Programas informáticos. Aprendizaje. 


\section{Introdução}

A incorporação de novas tecnologias no processo de ensino-aprendizagem é uma realidade cada vez mais presente nas salas de aula. Aumento do interesse dos alunos, facilidade na demonstração de conteúdos, minimização de custos com materiais, sem contar o alcance globalizado quando conectada à internet, são algumas das vantagens advindas do uso dessas novas tecnologias(1).

Quando se trata de ensino superior, entretanto, poucas tecnologias têm sido incorporadas em substituição às práticas laboratoriais que, muitas vezes, demandam o consumo de insumos e equipamentos caros. Esse fato pode estar relacionado à complexidade do desenvolvimento de softwares educacionais. Tais sistemas devem atender às necessidades pedagógicas, como por exemplo, não apresentar erros conceituais, e ao mesmo tempo, necessitam de profissionais da área de informática para traspor o que acontece no mundo real de forma sistematizada para o ambiente virtual $(2,3)$.

Cremes e loções são os principais veículos de produtos cosméticos e dermatológicos, sendo tema relevante para estudantes da área farmacêutica, química e cosmética. A formulação desses produtos é um processo laborioso, uma vez que depende de uma enorme quantidade de informações sobre os componentes que os constituem. Concentração de uso, solubilidade, estabilidade térmica, faixa de $\mathrm{pH}$, funções exercidas, carga residual, necessidade de antioxidantes e as incompatibilidades químicas, físicas e físico-químicas de todos os componentes da fórmula devem ser considerados no processo de formulação(4,5). O aprendizado das propriedades dos ingredientes e a correlação entre todos esses fatores demandam certo esforço, geralmente levando a um processo empírico de formulação ou à simples reprodução de fórmulas prontas que pouco contribuem no processo de ensino-aprendizagem.

Nesse contexto, a criação de um sistema de informação (SI) que armazena e processa as informações sobre os ingredientes de cremes e loções é de grande valor para otimizar o ensino desse conteúdo. Além disso, também pode contribuir para reduzir seu custo, uma vez que o usuário irá cometer a maioria dos erros no ambiente virtual sem o dispêndio de materiais.

O objetivo deste trabalho foi desenvolver um sistema Web direcionado a estudantes, profissionais e pesquisadores que tenha a aplicação como ferramenta virtual de ensino-aprendizagem no desenvolvimento de formulação de cremes e loções de uso cosmético e dermatológico.

\section{Metodologia}

Requisitos e Funcionalidades do Sistema

O desenvolvimento do software Formula ${ }^{\circledR}$ iniciou-se com a definição dos requisitos e funcionalidades mínimas que o sistema deveria apresentar, sendo eles: (i) armazenar em um banco de dados informações como funções e propriedades de ingredientes que normalmente são utilizados na criação de emulsões; (ii) possuir mecanismo que permitisse escolher esses ingredientes formando assim uma fórmula de emulsão; (iii) executar a validação da fórmula retornando posteriormente um resultado positivo ou negativo, (iv) gerar o método de preparo com a fórmula criada; (v) realizar o acompanhamento do processo de criação de fórmulas pelos usuários. Em seguida, foram definidos os perfis de usuários do sistema: (i) usuário comum, (ii) professor e (iii) administrador. O diagrama de caso de uso (Figura 1) apresenta as interações dos atores e as funcionalidades do sistema prédefinidas.

Figura 1: Diagrama de caso de uso.

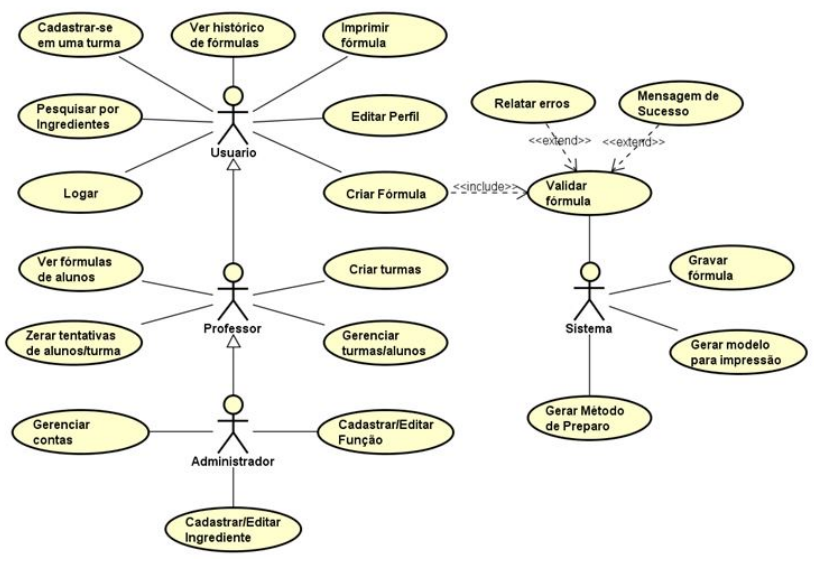

Desenvolvimento do Software Formula®

Após a definição dos requisitos e funcionalidades, foram criados diagramas de Linguagem de Modelagem Unificada (Unified Modeling Language - UML) com o auxílio do software Astah Community 7.0.0. O diagrama de classes mostra os atributos e os principais métodos necessários para as classes do sistema (Apêndice A) e o diagrama de sequência demonstra como o processo de criação de uma fórmula é realizado (Apêndice B).

Na sequência, deu-se início à construção do sistema Web, sendo que um dos primeiros passos foi encontrar um framework para servir de base no desenvolvimento. Aquele que mais atendeu às necessidades do projeto foi o framework PHP Laravel juntamente com o framework front-end Bootstrap. Para a criação do banco de dados e acompanhamento das informações gravadas foi utilizado o aplicativo MySQL Workbench 6.2 CE. Para utilizar os serviços MySQL e Hypertext Preprocessor (Preprocessador de Hipertexto - PHP), o WampServer 2.4 auxiliou nessa questão. Já o programa JetBrains PhpStorm 2016.1.1 ficou responsável pela codificação das linguagens utilizadas, sendo elas a linguagem de marcação HyperText Markup Language (Linguagem de Marcação de Hipertexto - HTML) para montagem da estrutura, Cascading Style Sheet (Folha de Estilo em Cascata - CSS) para a estilização, 
junto com JavaScript e a biblioteca jQuery.

O sistema está disponível no seguinte endereço eletrônico: http://200.195.138.66/emulsao/public/

\section{Resultados e discussão}

\section{Requisitos e Funcionalidades do Sistema}

$\mathrm{Na}$ área educacional nota-se um aumento no uso de SI's devido ao grande benefício que trazem para o aprendizado. Para a criação de um software educacional de qualidade foi necessário o envolvimento de profissionais da área de desenvolvimento e da área em que o software se destina, além dos utilizadores que tem um papel fundamental na especificação e validação dos requisitos coletados(6). Nesse sentido, para definir bem as funcionalidades que o sistema devia ter foi preciso fazer uma especificação detalhada dos requisitos(7).

A funcionalidade essencial do software Formula ${ }^{\circledR}$ encontra-se no mecanismo que permite a criação de fórmulas de cremes e loções a partir da escolha dos componentes da formulação que, por sua vez, tem suas propriedades armazenadas em um banco de dados do próprio sistema, conforme mostrado na Figura 2. Com as regras predeterminadas pelos desenvolvedores, o sistema valida essa fórmula retornando posteriormente um resultado positivo ou negativo, como pode ser visto respectivamente nas Figuras 3 e 4 .

Figura 2: Tela para criação de fórmula.

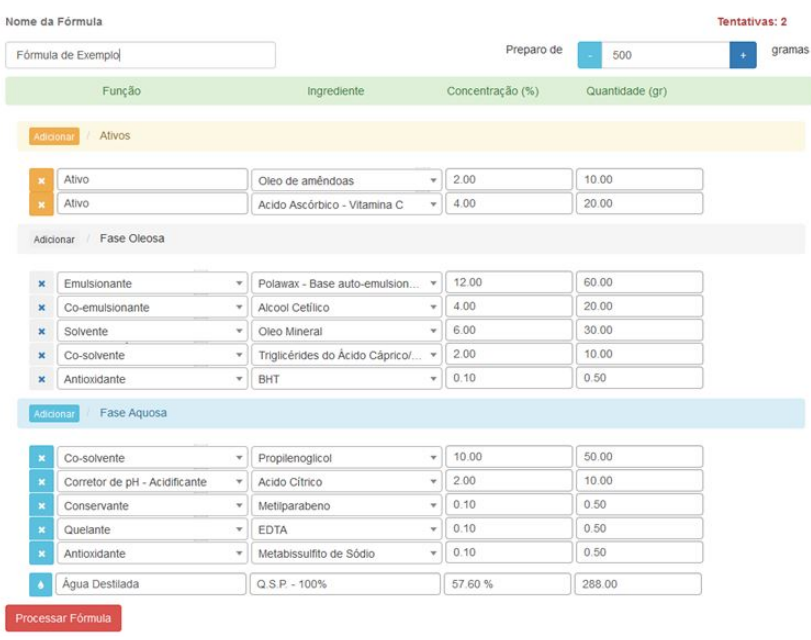

Figura 3: Exemplo de resultado positivo de uma fórmula.

Fórmula processada

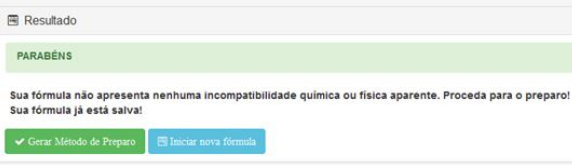

Figura 4: Exemplo de resultado negativo de uma fórmula.

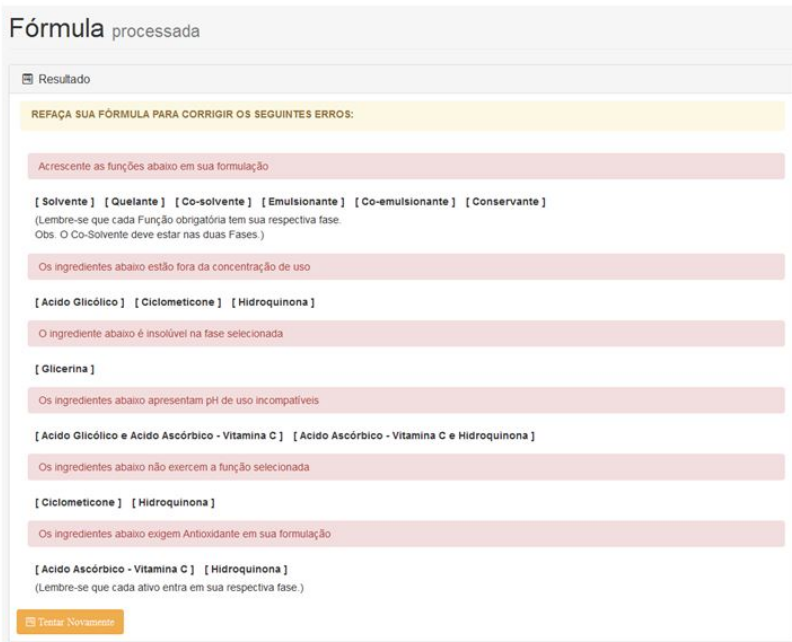

A validação da fórmula criada pelo usuário é realizada levando em consideração quinze regras relacionadas à formulação de cremes e loções. A concentração de uso, solubilidade, estabilidade térmica, faixa de $\mathrm{pH}$, funções exercidas, carga residual, necessidade de antioxidantes e as incompatibilidades de todos os componentes da fórmula são levados em consideração no processo de validação da mesma, conforme mostra o Quadro 1. Se o sistema encontra algum erro aparente, o mesmo é mostrado; mas, em caso de aprovação, a fórmula é salva e o sistema gera o método de preparo com a fórmula criada (Figura 5).

Quadro 1: Regras para validação das formulações.

\begin{tabular}{|c|c|}
\hline 1 & A fórmula não deve conter mais de um emulsionante. \\
\hline 2 & $\begin{array}{l}\text { A fórmula deve conter pelo menos todas as sete funções de ingrediente obrigatórias: } \\
\text { emulsionante, co-emulsionante, solvente F.O., co-solvente F.O., co-solvente F.A., } \\
\text { conservante e quelante. }\end{array}$ \\
\hline 3 & Os ingredientes devem estar dentro da concentração de uso definida no cadastro. \\
\hline 4 & A fórmula não deve conter ingredientes quimicamente incompativeis. \\
\hline 5 & $\begin{array}{l}\text { Os ingredientes devem estar na Fase Aquosa ou Oleosa de acordo com sua } \\
\text { solubilidade. }\end{array}$ \\
\hline 6 & Os ingredientes da fórmula não devem apresentar pH de uso incompatíveis. \\
\hline 7 & Os ingredientes devem exercer a função selecionada na fórmula. \\
\hline 8 & $\begin{array}{l}\text { Se um ingrediente for selecionado para atuar como ativo na fórmula ele } \\
\text { obrigatoriamente deve ser um ativo. }\end{array}$ \\
\hline 9 & $\begin{array}{l}\text { Se ingredientes que precisem de antioxidante estiverem na fórmula a mesma deve } \\
\text { conter pelo menos um antioxidante na fase em que o ingrediente se encontra. }\end{array}$ \\
\hline 10 & $\begin{array}{l}\text { Caso ingredientes que exigem correção de pH estiverem na fórmula, a mesma deve } \\
\text { conter um corretor de pH, e não mais que um. }\end{array}$ \\
\hline 11 & Antioxidantes sem necessidade não devem aparecer na fórmula. \\
\hline 12 & A fórmula não deve conter corretor de pH sem uso na Fase Aquosa. \\
\hline 13 & A Fase Oleosa nunca deve conter corretores de pH. \\
\hline 14 & Um ingrediente não pode aparecer mais de uma vez na fórmula. \\
\hline 15 & $\begin{array}{l}\text { A Fase Oleosa possui limite proporcional de } 40 \% \text {, consequentemente a Fase Aquosa } \\
\text { pode ultrapassar } 60 \% \text {. }\end{array}$ \\
\hline
\end{tabular}


Figura 5: Exemplo de fórmula e método de preparo para impressão.

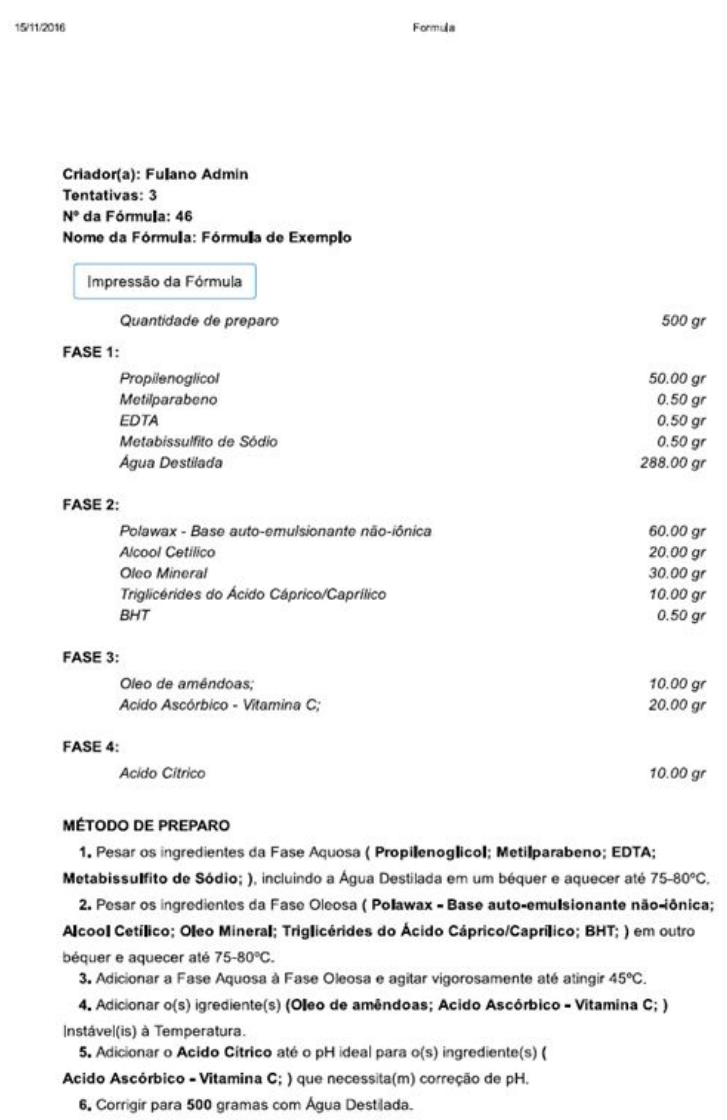

O sistema elabora o método de preparo ajustando a ordem de adição dos ingredientes de acordo com a solubilidade, estabilidade térmica e função dos mesmos. Também calcula a quantidade dos ingredientes a ser pesada de acordo com as porcentagens escolhidas pelo usuário e a quantidade de produto a ser preparada. O software não utiliza o sistema de classificação denominado Equilíbrio Hidrófilo-Lipófilo (EHL) para executar a validação de fórmulas, devido a complexidade e as limitações dessa metodologia. Mas os valores de EHL dos emulsionantes podem ser encontrados na ficha técnica disponibilizada no cadastro de ingredientes.

Foram definidos três tipos de usuários para o sistema: usuário comum, professor e administrador, conforme mostrado no Diagrama de Caso de Uso (Figura 1). Os usuários comuns no sistema podem realizar o processo de criação de fórmulas inúmeras vezes, pesquisar por ingredientes cadastrados (Figura 6), visualizar seu histórico de fórmulas, sendo que cada fórmula recebe uma cor de acordo com seu número de tentativas, e cadastrar-se em uma turma (Figura 7).
Figura 6: Tela para busca de ingredientes.

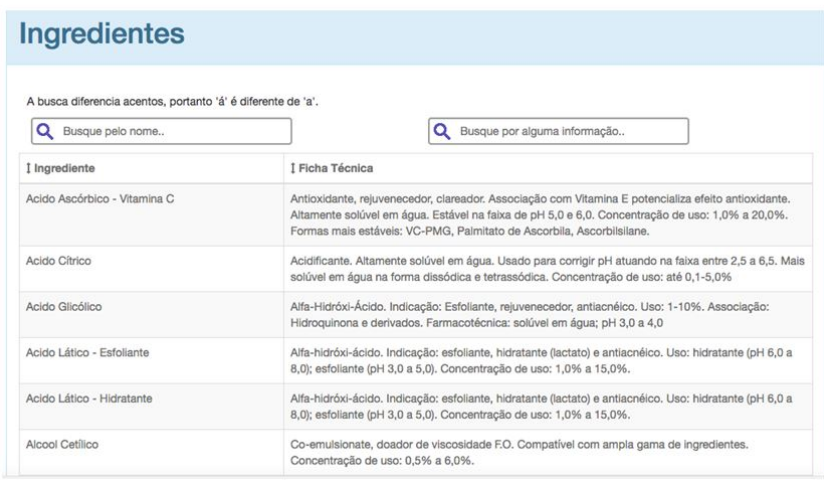

Figura 7: Exemplo de um histórico de fórmulas.

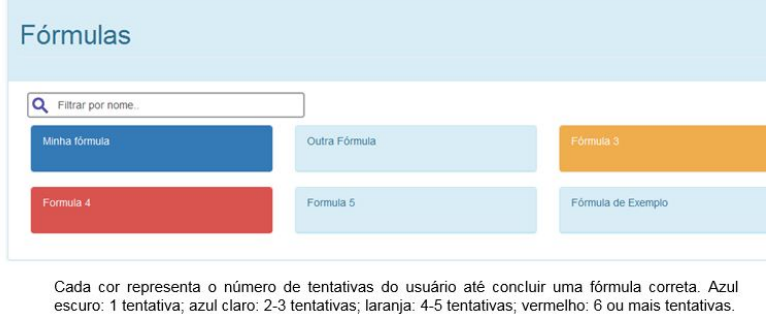

Os usuários definidos como professor no sistema também podem criar turmas e gerenciá-las, além de visualizar os alunos cadastrados nelas (Figura 8). As respectivas fórmulas de cada aluno também podem ser vistas pelo professor. Ele ainda pode zerar o contador de tentativas de cada aluno ou da turma inteira com o objetivo de iniciar uma nova atividade e avaliar o número de vezes que cada aluno refez a fórmula até obter sucesso na formulação.

Figura 8: Cadastro em uma Turma
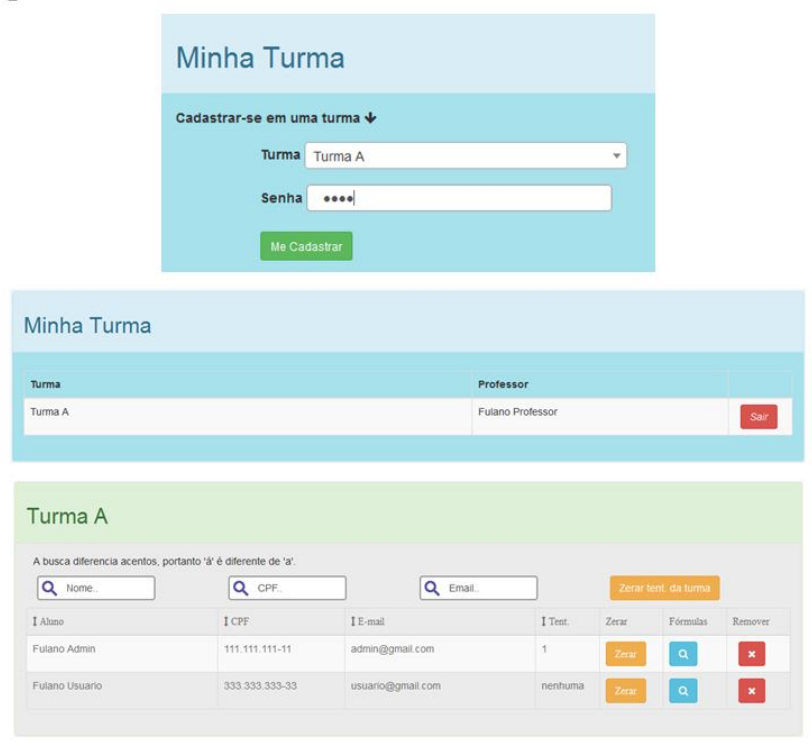
Por fim, o administrador, que além de ter acesso as mesmas tarefas dos demais tipos de usuários, pode cadastrar e editar ingredientes e funções, como no exemplo da Figura 9 e gerenciar as contas dos usuários.

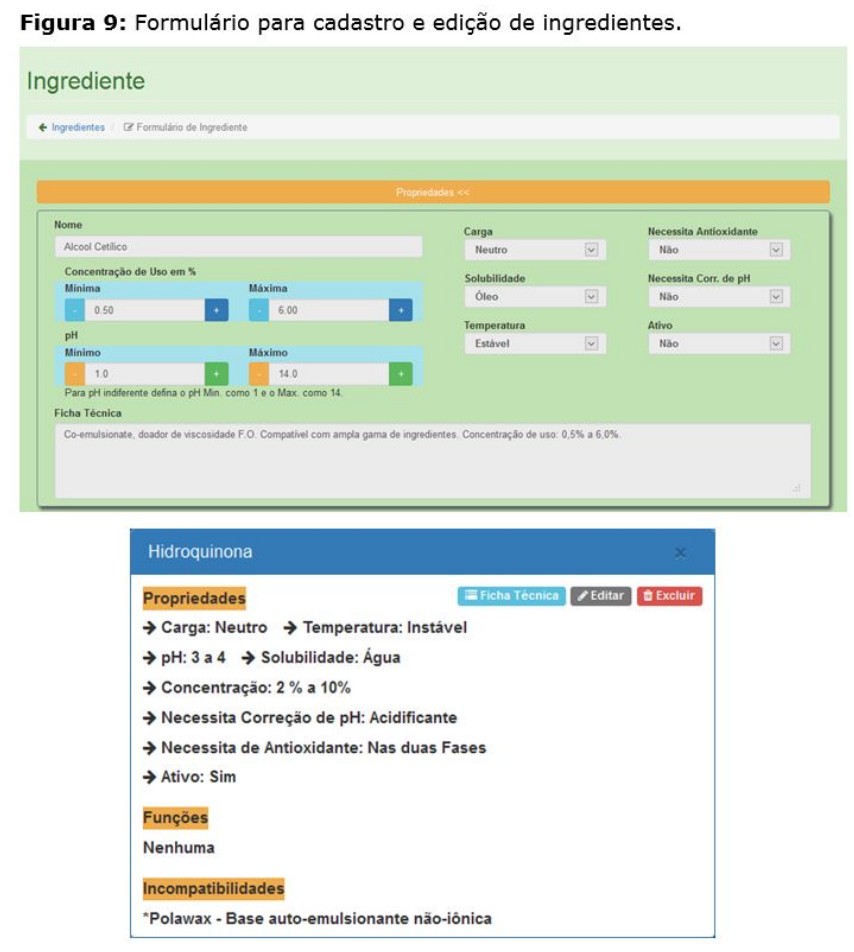

Ao criar uma conta, o usuário é automaticamente definido como usuário comum. Para que o mesmo possa atuar com outra função no sistema será preciso que o administrador altere seu tipo. É normal um usuário esquecer sua senha e para resolver esse problema o sistema permite que um administrador altere a senha desse usuário para uma senha padrão. Para que isso aconteça, o usuário precisa informar o acontecido ao administrador via e-mail, enviando seus dados de usuário, como e-mail e CPF. Após a alteração da senha o usuário já poderá acessar novamente o sistema e alterar a senha para uma pessoal de sua preferência.

No sistema o usuário pode realizar buscas por ingredientes (Figura 6), de modo a agilizar ainda mais a criação de uma fórmula. Para tanto, basta apenas que o usuário inicie a digitação do nome do ingrediente no campo apropriado e os ingredientes vão sendo automaticamente filtrados. Sem esta ferramenta seria necessária a busca de informações sobre determinada matéria-prima em vários livros ou sites.

Cada fórmula contém um número único seriado para garantir a autenticidade do processo de formulação. Caso o professor queira a fórmula impressa o aluno não terá sucesso em alterá-la, pois o professor pode comparar a fórmula impressa com a do sistema por meio desse número. E, caso as características não forem iguais, significa que o aluno fez alterações na fórmula para a impressão.
Os professores também podem avaliar os alunos por meio do sistema com o auxílio do contador de tentativas. Com ele o professor pode saber a quantidade de tentativas que cada aluno precisou para realizar uma determinada fórmula. Caso o professor queira passar uma determinada tarefa para a resolução dos alunos, ele pode zerar o contador de tentativas de cada um, fazendo com que todos sejam avaliados igualmente, pois cada aluno pode estar com um valor de tentativas diferente.

O sistema traz formulários simples e fáceis para o cadastro de ingredientes e funções, de modo que o usuário administrador não precisa de qualquer treinamento para realizar qualquer um desses cadastros (Figura 9). O número de funções e ingredientes a serem cadastrados é ilimitado. $\mathrm{O}$ mesmo vale para as outras funcionalidades do sistema, que são bastante autoexplicativas e o usuário consegue realizálas facilmente.

\section{Desenvolvimento do Software Formula ${ }^{\circledR}$}

Após a modelagem dos diagramas pode-se dar início à criação do sistema em si. Os diagramas atuam como uma base de desenvolvimento na qual se pode encontrar uma biblioteca de códigos, classes e funções que ajudam na criação de sistemas que são moldados para uma determinada área(8).

Muitos desenvolvedores utilizam frameworks para criação de sistemas Web. O Laravel é um framework PHP criado por Taylor Otwell, em 2011(9), para conter algumas funcionalidades que o framework CodeIgniter até então não possuía. Com isso, um novo conjunto de ferramentas para o desenvolvimento de sistemas Web permitiu maior aceitação entre os desenvolvedores do mundo inteiro. Além disso, o motivo dessa popularidade é devido a sua facilidade de aprendizado com sua documentação, seus recursos, sua comunidade e a sua boa estabilidade(10).

O processo de desenvolvimento de um sistema que utilize o Laravel é padronizado, com isso ele já processa algumas tarefas lógicas automaticamente, sem a atuação do programador. Dessa forma, permitindo ao desenvolvedor focar na aplicação da lógica de negócio no sistema(11).

Para que o conteúdo presente nas páginas fique organizado, é preciso utilizar alguma linguagem que estruture esse conteúdo. A linguagem de marcação HTML criada em 1991 estrutura esses documentos. Para criar páginas Web, o HTML possui uma sintaxe própria que demarca seu conteúdo por meio de tag's simples e, devido a sua simplicidade, qualquer editor de texto pode manipular seu código sem a necessidade de usar algum Integrated Development Environment (Ambiente de Desenvolvimento Integrado IDE) específico. Para a visualização do resultado obtido basta salvar o documento em formato .html e abri-lo com algum navegador(12).

A maioria dos sites na Web precisa trabalhar de forma dinâmica para que seu conteúdo seja atualizado a cada momento, de modo que utilizando apenas uma linguagem 
estática, como o HTML, isso acaba se tornando inviável, principalmente se o site possui muitas páginas. Esse problema pode ser resolvido com o uso da linguagem de programação PHP ypertext Preprocessor (Pré-processador de Hipertexto - PHP), que pode ser mesclado com o HTML. O código é executado no servidor gerando a página em HTML vista pelo usuário, sendo que dentre as muitas vantagens do PHP uma delas é que o site pode ser dividido em várias partes, podendo ser montado de acordo com cada página(13). Dessa forma, a criação e edição das páginas se torna uma tarefa fácil, uma vez que cada parte pode ser aproveitada por várias páginas, sem a necessidade de repetição de código, além de trabalhar com a orientação a objetos e suporte a vários tipos de banco de dados.

Um site ideal deve ter uma boa aparência e o framework front-end Bootstrap pode proporcionar essa ajuda para a criação de layouts. Esse tipo de framework funciona como um esqueleto que se adapta às necessidades do site. Seu código opensource contém plug-ins jQuery, arquivos Cascading Style Sheet (Folha de Estilo em Cascata - CSS) prontos para estilização e vários outros componentes disponíveis que facilitam a modelagem da página(14). Por utilizar um sistema de grades ele é facilmente dividido em até doze colunas que podem ser definidas para se adequar a diferentes resoluções de tela. Dessa forma, o usuário terá sempre a mesma experiência de uso.

A estilização CSS, por sua vez, se destina a páginas Web. Sua utilização reduz o tempo de criação de uma página, dando controle total do layout. Apenas uma folha de estilos pode controlar o layout de várias páginas. Logo, por esse motivo, é vantajoso utilizar o CSS em projetos Web(15).

Para trabalhar com toda a codificação das linguagens presentes em um projeto Web, é preciso utilizar algum tipo de software que possibilite a manipulação desses códigos. Para isso, existem os IDE, que são um ambiente de desenvolvimento. Algumas das principais vantagens dos IDE é a de compilar várias bibliotecas de linguagem e devido a capacidade de identificar uma determinada linguagem os debuggers presentes no software podem apontar alguns erros que podem vir a aparecer no código(16). Muitos IDEs também autocompletam algumas palavras e até mesmo funções naturais da linguagem, facilitando o desenvolvimento para o programador. Dessa forma, visto o benefício de usar um IDE, a empresa JetBrains desenvolveu o PhpStorm. Este IDE é ótimo para trabalhar com os frameworks mais conhecidos atualmente, pois o PhpStorm compreende e analisa o código e sua estrutura, colorizando o mesmo e, assim, facilitando a visualização para o desenvolvedor e o ajudando a escrever um código limpo. Além de possuir inúmeras ferramentas, o PhpStorm é bastante seguro, como, por exemplo, a função que salva instantaneamente seu código após qualquer alteração feita nele, evitando perdas inesperadas(17).

Em muitos casos os SI's precisam armazenar dados para serem ocupados futuramente. Para isso, é necessário que haja um Sistema Gerenciador de Banco de Dados (SGBD), e o MY SQL Structured Query Language (Linguagem de Consulta Estruturada - MySQL) pode ser uma opção para realizar essa tarefa, pois contém muitos recursos que os outros grandes SGBD também possuem. Porém, o MySQL tem uma grande vantagem sobre os outros concorrentes, pois além de ter os mesmos recursos e ser mais fácil de programar, ele é gratuito(18). Uma ferramenta visual que pode ser usada para trabalhar com o banco MySQL é o software MySQL Workbench. Ele permite criar e gerenciar visualmente um banco de dados, podendo fazer modelos complexos além de oferecer recursos para tratar a engenharia reversa(19).

No desenvolvimento de aplicações Web muitas vezes é necessário o uso de um servidor, além dos serviços PHP e de um banco de dados. Para facilitar o uso desses recursos o WampServer traz todos os três no mesmo software, tendo o Apache como servidor e o banco de dados MySQL. Com ele o usuário não precisa se preocupar com as configurações de um servidor, apenas executá-lo para ter acesso a todos os seus serviços $(20)$.

\section{Conclusão}

Com o uso do software Formula ${ }^{\circledR}$ o processo de criação de uma fórmula de emulsão se tornou uma tarefa muito mais fácil, pois o sistema traz uma estrutura simples, além de diminuir consideravelmente o tempo de análise de erros de uma fórmula pelo usuário. Em suma, a incorporação de novas tecnologias no processo de ensino-aprendizagem se tornou uma tarefa imprescindível para acompanhar a evolução dos meios de comunicação propiciada pela internet e, mais especificamente, pelo compartilhamento de informações de qualquer indivíduo, quebrando, assim, as barreiras de tempo e espaço.

\section{Referências}

1. Giraffa LM. Uma odisseia no ciberespaço: O software educacional dos tutoriais aos mundos virtuais. RBIE. 2009;17(1):20-30.

2. Benitti FBV, Seara EFR, Schlindwein LM. Processo de Desenvolvimento de Software Educacional: proposta e experimentação. RENOTE - CINTED-UFRGS. 2005;3(1).

3. LIMA MM, Lima AR, Monteiro ACC, Cavalcante Júnior EH, Gomes, LQL. Uma Revisão Sistemática da Literatura dos Processos de Desenvolvimento de Software Educativo. Anais do $23^{\circ}$ Simpósio Brasileiro de Informática na Educação (SBIE 2012), ISSN 2316-6533. Rio de Janeiro, 2012.

4. AULTON ME. Delineamento de Formas Farmacêuticas. Artmed, $2^{\mathrm{a}}$ ed., Porto Alegre, 2005.

5. Allen LV, Popovich NG, Ansel H. Formas Farmacêuticas e Sistemas de Liberação de Fármacos. Artmed. $9^{a}$ ed., Porto Alegre, 2013.

6. Costa AP, Costa EB. Contributos para o Desenvolvimento de Software Educativo tendo por base Processos Centrados no Utilizador. EM TEIAl Revista de Educação Matemática e Tecnológica Iberoamericana. 2013;4(2). 
7. Giraffa L, Marczak S, Prikladnicki R. PDS-E: Em direção a um processo para desenvolvimento de Software Educacional. In:Anais do Workshop de Informática na Escola. 2005.

8. Minetto EL. Frameworks para Desenvolvimento em PHP. São Paulo: Novatec, 2007.

9. Surguy M. History of Laravel PHP framework, Eloquence emerging. 2013. [cited 2016 august 25]. Available from: < http://maxoffsky.com/code-blog/history-oflaravel-php-framework-eloquence-emerging/>.

10. Bezerra PT, Schimiguel J. Desenvolvimento de Aplicações Mobile Cross-Platform utilizando Phonegap, 2016.

11. Yu HR. Design and implementation of web based on Laravel framework. 2014 International Conference on Computer Science and Electronic Technology (ICCSET 2014). ShenZhen: Atlantis Press 2014.

12. Pacievitch Y. HTML. InfoEscola. [cited 2016 september 08]. Available from: < http://www.infoescola. com/informatica /html>

13. Achour M, Betz F, Dovgal A, Lopes N, Magnusson H, Richter G et al. Manual do PHP. [cited 2016 september 09]. Available from: < http://php.net/manual/pt_BR/>.

14. Araújo AHM. Web Design Responsivo, 2015.

15. Jobstraibizer F. Criação de sites com o CSS: Desenvolva páginas Web mais leves e dinâmicas em menos tempo. 1. ed. Digerati Books, 2009.

16. Novaes R. O que é e para que serve IDE?. PSafe Blog. [cited 2016 september 09]. Available from < http://www.psafe.com/blog/o-que-serve-ide/ >.

17. Jetbrains. PhpStorm: Lightning-smart PHP IDE. (C) 2000-2016 JetBrains. [cited 2016 september 09]. Available from < https://www.jetbrains.com/phpstorm/ $>$.

18. Veiga DM, Dellê FJ. Desenvolvimento de Sistema Web para Gerenciamento de Bancas Avaliadoras de Trabalho de Conclusão de Curso, 2015.

19. Oracle Corporation. MySQL Workbench. MySQL. [cited 2016 september 06]. Available from: < http://www. mysql. com/products /workbench/ $>$.

20. Bourdon R. WampServer: a Windows web development environment. WampServer. [cited 2016 september 09]. Available from: < http://www.wampserver.com/en/>.

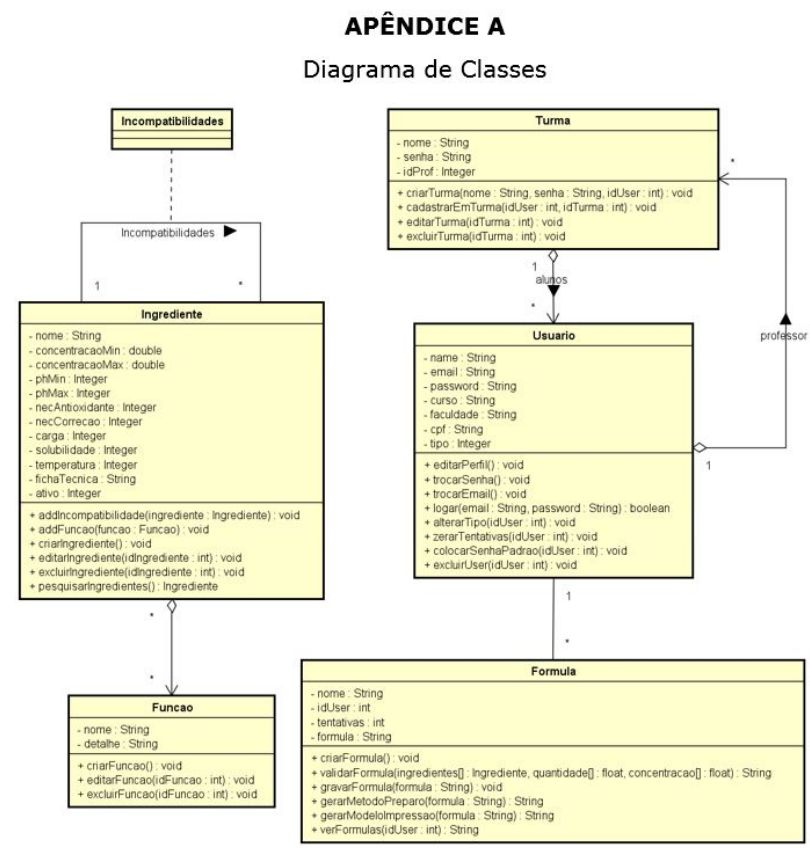

APÊNDICE B

Diagrama de sequência

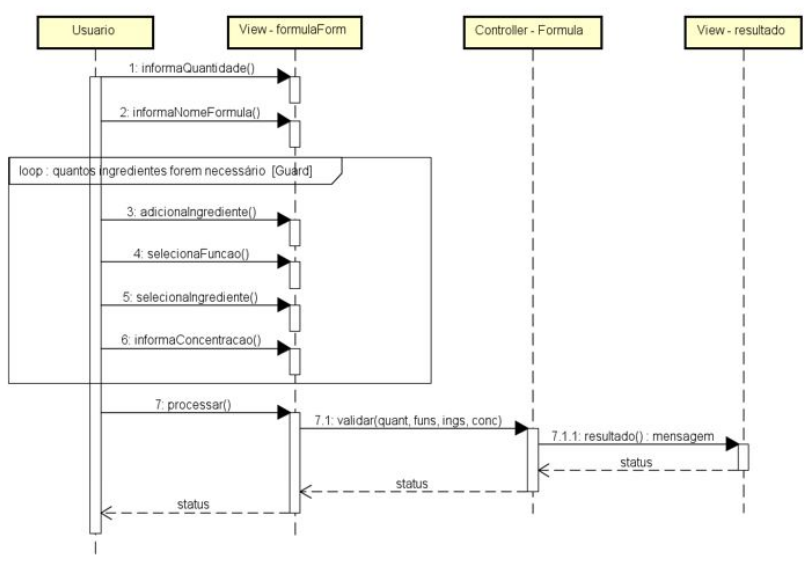

